\title{
AVALIAÇÃO DAS PREFERÊNCIAS DOS CONSUMIDORES DE PESCADO DO ESTADO DO TOCANTINS ATRAVÉS DE PESQUISA DE CAMPO REALIZADA NO SEMINÁRIO CAIU NA REDE É LUCRO
}

\author{
FLORES, R. M. V.*; CHICRALA, P. M. \& SOARES, S. S. \\ Embrapa Pesca e Aquicultura \\ *Corresponding author: roberto.valladao@embrapa.br
}

\begin{abstract}
Flores, R. M. V.; Chicrala, P. M. \& Soares, S. S. (2014) Preference evaluation of fish consumer's from Tocantins State, through field research during the "Caiu na rede é lucro" seminar. Braz. J. Aquat. Sci. Technol. 18(1): 121-129. eISSN 1983-9057. DOI: 10.14210/bjast.v18n1.p121-129 Due to the large growth of the fishery sector in recent years and the strong upward trend of production and consumption for the future, it is necessary to cover a gap in the literature on fish consumption and evaluate consumer preferences. For this, the present study aims to identify these preferences to the state of Tocantins, in northern Brazil, conducting field research in the national seminar "Caiu na Rede é Lucro". The event was promoted by the system FAET/SENAR (Federação da Agricultura e Pecuária do Estado do Tocantins), Palmas, capital of the state of Tocantins and it was part of the works of FAET for the 'Fish Multiplication Year'. The seminar was attended by approximately 300 people and some of these professionals work in fishing or aquaculture. Besides these professionals, the event also featured many interested in this subject and regular consumers of fish. To collect the desired data, a questionnaire was delivered to all participants of the seminar. As a result, the species of fish preferred for consumption were peacock bass and snapper. The study also investigated the preferences of conservation, presentation and local for purchase of fish. Fresh fish was the method of conservation chosen by most people, while whole fish was the preferred presentation form. With respect to purchasing local, the market was the most indicated alternative used by consumers.
\end{abstract}

Keywords: Seafood consumption, Tocantins state, Consumption habits.

\section{INTRODUÇÃO}

Além de projeções da Food and Agriculture Organization of the United Nations - FAO (2012) sobre o aumento populacional e da produção agrícola, onde calcula-se que a necessidade de incremento da produção agrícola até 2050 seja por volta de $60 \%$, há também evidências, indicadas por Roppa (2009), de que a demanda por proteína animal no mundo deve continuar apresentando acentuado crescimento, assim como nos últimos 40 anos, com o consumo anual per capita mundial de carnes passando de 23kg em 1961 para 46,6kg em 2009.

Devido a essas projeções e ao fato de a proteína animal mais produzida no mundo atualmente ser a carne de pescado, alcançando 154 milhões de toneladas em 2011 (FAO, 2012), o consumo de pescado vem sendo apontado como uma das possíveis soluções para o aumento da demanda mundial por alimentos. Para o Brasil, há tendência de grande aumento na produção aquícola e estabilidade na produção pesqueira (Sidonio et al., 2012). Surge então a necessidade de analisar o mercado interno brasileiro e de se obter maior entendimento sobre as preferências do consumidor (Maciel, 2011). Essa necessidade fica mais evidente, pois há grande diversidade de fatores sociais, culturais e econômicos que influenciam o consumo do pescado no Brasil (Ostrensky et al., 2008).

Nesse sentido, torna-se necessário cobrir uma lacuna na literatura sobre consumo de pescado e avaliar as preferências do consumidor no que se refere a locais de compra, espécies preferidas, formas de apresentação e tipos de conservação (Sonoda, 2007). Para isso, o presente trabalho buscou caracterizar o consumidor de pescado no estado do Tocantins, presente na região Norte do Brasil, realizando pesquisa de campo no seminário nacional "Caiu na Rede é Lucro". O evento foi promovido pelo sistema FAET/SENAR (Federação da Agricultura e Pecuária do Estado do Tocantins/Serviço Nacional de Aprendizagem Rural), no dia 26 de março de 2012 em Palmas, capital do estado do Tocantins e fez parte das ações da FAET para o considerado Ano da Multiplicação dos Peixes.

\section{MATERIAIS E MÉTODOS}

O seminário contou com a presença de aproximadamente 300 pessoas. Desses participantes, muitos são profissionais da área de pesca ou aquicultura. Além dos profissionais, o evento também contou com muitos interessados pelo assunto e consumidores de pescado de modo geral. Para a coleta dos dados desejados foram entregues questionários a todos os participantes do seminário.

Nesse questionário, alguns pontos importantes foram observados para garantir qualidade das respostas obtidas, maior número de questões respondidas e maior possibilidade de análise das respostas por estratificação dos indivíduos. As questões aplicadas podem ser encontradas no Apêndice 1 . 
A aplicação dos questionários foi realizada através do autopreenchimento. Assim, os participantes responderam as questões de próprio punho durante a realização do evento. Esta metodologia possui vantagens e desvantagens. As principais vantagens são o tempo ganho na coleta das informações, o maior número de questionários aplicados e o fato de o participante se sentir mais a vontade para responder perguntas de cunho pessoal como o nível de escolaridade, por exemplo. Do lado das desvantagens tem-se o fato do possível não entendimento das questões aplicadas (que em muitos casos resulta em variáveis não aproveitadas na pesquisa) e o fato de não ser eficiente um questionário que colete muitas variáveis e desestimule o indivíduo a responder todas as questões.

Desta forma, os questionários aplicados tiveram 14 questões, todas presentes na frente de uma única folha de papel. Na maioria das questões, priorizou-se o uso de alternativas de resposta, ao invés do uso de questões abertas, que tem as vantagens de mais fácil entendimento para coleta e agrupamento das variáveis e maior rapidez no preenchimento. Por outro lado, questões com alternativas podem direcionar a intenção de resposta de alguns indivíduos.

Durante o evento, 162 participantes responderam aos questionários da pesquisa, de um público total de, aproximadamente, 300 pessoas. Baseado nesses números, a pesquisa possui uma margem de erro de $4,39 \%$ para mais ou para menos, considerando que os dados seguem uma distribuição normal, com $90 \%$ de intervalo de confiança.

\section{RESULTADOS}

Participantes de quatro diferentes unidades federativas responderam os questionários da pesquisa apresentada. Ao serem questionados em qual unidade federativa os participantes residem, foram obtidos como resposta os estados de Mato Grosso, Tocantins, Santa Catarina e Amazonas. Como esperado, pelo fato de o evento ter ocorrido em Palmas, capital do estado do Tocantins, a grande maioria dos participantes re- side no estado que abrigou o seminário. Assim, 97\% dos participantes responderam residir no Tocantins. Os demais estados, tiveram uma representação de aproximadamente $1 \%$ cada um.

Com relação ao município de residência, 45,6\% dos participantes responderam Palmas. Além disso, mais 28 municípios possuem representantes na pesquisa. Os municípios de Colmeia, Almas, Gurupi e Porto Nacional, todos do Tocantins, tiveram uma participação de $16 \%, 3,8 \%, 3,2 \%$ e 3,2\%, respectivamente, enquanto os demais tiveram uma participação menor que $3 \%$.

Dos participantes do seminário, a maioria é do sexo masculino $(74,51 \%)$ : para cada participante mulher $(25,49 \%)$, havia três participantes homens.

Um dos pontos mais importantes ao se avaliar as respostas dos participantes é verificar o nível de escolaridade. Assim, a Tabela 1 apresenta a distribuição do nível de escolaridade de todos participantes que responderam a pesquisa, dos participantes que estudam ou trabalham na área de pesca ou aquicultura e dos participantes que não estudam ou trabalham na área.

Esta análise é importante, pois, dependendo do nível de escolaridade, os participantes podem ter diferentes demandas e necessidades perante 0 consumo de pescado e tecnologias e cursos. Como pode ser visto na Tabela 1, a maioria dos participantes possui ao menos o ensino médio completo, o que dá mais consistência aos resultados obtidos. Além disso, os participantes que não são profissionais da área de pesca ou aquicultura possuem, na média, nível de escolaridade mais alto comparado aos profissionais.

Com relação à área de atuação, os participantes foram questionados se trabalham ou estudam na área de pesca ou aquicultura e, se trabalham, com quais espécies. A pesquisa mostrou que $56 \%$ dos participantes não trabalham ou estudam na área. $O$ fato de alguém ser um profissional da área pode ter influência nos demais resultados. Desta forma, ao longo da apresentação dos resultados deste trabalho, sempre serão consideradas as respostas de acordo com o fato de o participante trabalhar ou estudar na área.

Tabela 1 - Distribuição do nível de escolaridade dos participantes

\begin{tabular}{lccc}
\hline \hline $\begin{array}{l}\text { Nível de } \\
\text { escolaridade }\end{array}$ & $\begin{array}{c}\text { Todos } \\
\text { participantes }\end{array}$ & $\begin{array}{c}\text { Profissionais } \\
\text { da área }\end{array}$ & $\begin{array}{c}\text { Não } \\
\text { profissionais } \\
\text { da área }\end{array}$ \\
\hline Ensino Fundamental & $20,25 \%$ & $28,36 \%$ & $11,63 \%$ \\
Ensino Médio & $40,51 \%$ & $37,31 \%$ & $43,02 \%$ \\
Ensino Superior & $21,52 \%$ & $19,40 \%$ & $24,42 \%$ \\
Pós-graduação & $13,29 \%$ & $11,94 \%$ & $15,12 \%$ \\
Mestrado & $2,53 \%$ & $0,00 \%$ & $4,65 \%$ \\
Doutorado & $1,90 \%$ & $2,99 \%$ & $1,16 \%$ \\
\hline \hline
\end{tabular}




\section{Espécies de peixes preferidas para alimentação}

Os participantes da pesquisa foram questionados sobre a preferência de consumo de peixes. Assim, a Questão 3 dos questionários foi: "Qual é a espécie de peixe preferida para alimentação?". Assim, será analisada a preferência dos participantes para alguns extratos da pesquisa.

A pesquisa indica que, com $22,14 \%$, caranha é a espécie preferida para consumo entre os participantes dos cursos do seminário (tabela 2). Tucunaré, tambaqui e pintado, com $21,43 \%, 15,00 \%$ e $15,00 \%$, respectivamente, também foram espécies lembradas por um número significativo de pesquisados. Além destas espécies já citadas, pirarucu, piau, dourado, matrinxã, salmão, tambaú, tilápia, cachara, manpará, pacu piramutaba, surubim e tambatinga foram citadas pelos participantes.

A distribuição das espécies preferidas por gênero é apresentada na figura 1. No caso da preferência masculina, a caranha foi a espécie mais citada, assim como no gráfico anterior, mas com uma preferência maior ainda entre os homens. Já no caso das preferências femininas, o tucunaré é a espécie preferida com um terço das preferências. Vale ressaltar que a caranha que é a espécie preferida entre os homens praticamente não foi lembrada entre as mulheres.

A Tabela 3 apresenta as preferências por espécies de peixe, dado o nível de escolaridade. Como pode ser observado, caranha foi a espécie preferida entre os níveis de escolaridade mais baixos. Já entre os níveis mais altos, tucunaré e tambaqui foram as espécies mais citadas.

Por fim, é válido verificar como foi a distribuição das preferências entre os participantes do seminário que trabalham ou estudam pesca ou aquicultura (figu-

Tabela 2 - Distribuição das preferências de espécie de peixes para alimentação.

\begin{tabular}{lc}
\hline \hline \multicolumn{1}{c}{ Espécie } & $\%$ \\
\hline Caranha & $22,14 \%$ \\
Tucunaré & $21,43 \%$ \\
Pintado & $15,00 \%$ \\
Tambaqui & $15,00 \%$ \\
Pirarucu & $6,43 \%$ \\
Piau & $4,29 \%$ \\
Tilápia & $3,57 \%$ \\
Pacu & $2,14 \%$ \\
Surubim & $2,14 \%$ \\
Pacu caranha & $1,43 \%$ \\
Manpará & $1,43 \%$ \\
Dourado & $1,43 \%$ \\
Cachara & $0,71 \%$ \\
Matrinxã & $0,71 \%$ \\
Piramutaba & $0,71 \%$ \\
Salmão & $0,71 \%$ \\
Tambatinga & $0,71 \%$ \\
\hline \hline
\end{tabular}

ra 2). Para este grupo, além da caranha, tambaqui e tucunaré também foram as espécies preferidas.

\section{Forma de conservação de pescado mais comprada}

A Questão 5 dos questionários respondidos pelos participantes do seminário é "Em relação à forma de conservação, qual das opções é a mais comprada por você?". A figura 3 mostra que a grande maioria dos participantes aponta o pescado fresco como a forma de conservação de pescado mais comprada, com quase $70 \%$ das respostas. Pescado resfriado e pescado congelado foram, com $13,5 \%$ e $15 \%$, respectivamente, as formas de conservação com uma porcentagem também significativa na pesquisa. Por outro lado, pescado salgado, pescado defumado e pescado em conserva praticamente não foram selecionados na pesquisa, com menos de $1 \%$ cada um.

Quando se considera apenas as respostas dos profissionais da área, a distribuição das tecnologias demandadas segue conforme a figura 4 . Como pode ser visto, este gráfico muda pouco em relação à figura 3, mostrando que o fato de o participante ser um profissional da área de pesca ou aquicultura não tem

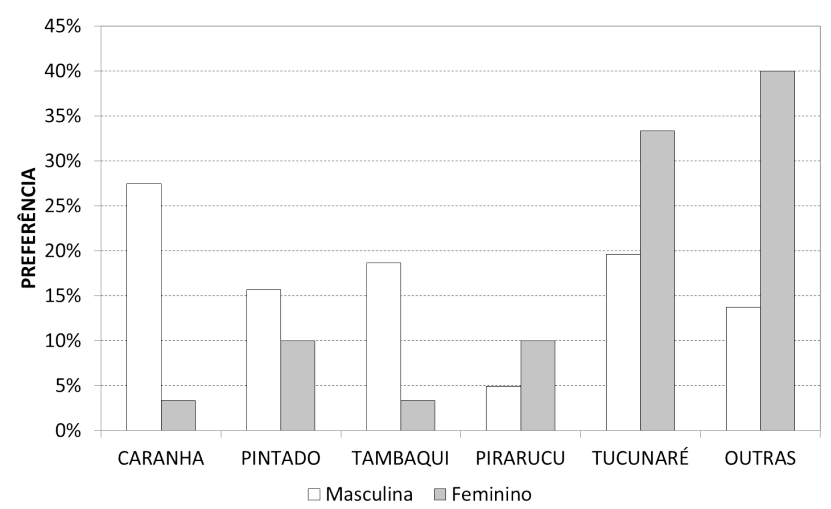

Figura 1 - Distribuição das preferências masculinas e femininas de espécie de peixes para alimentação

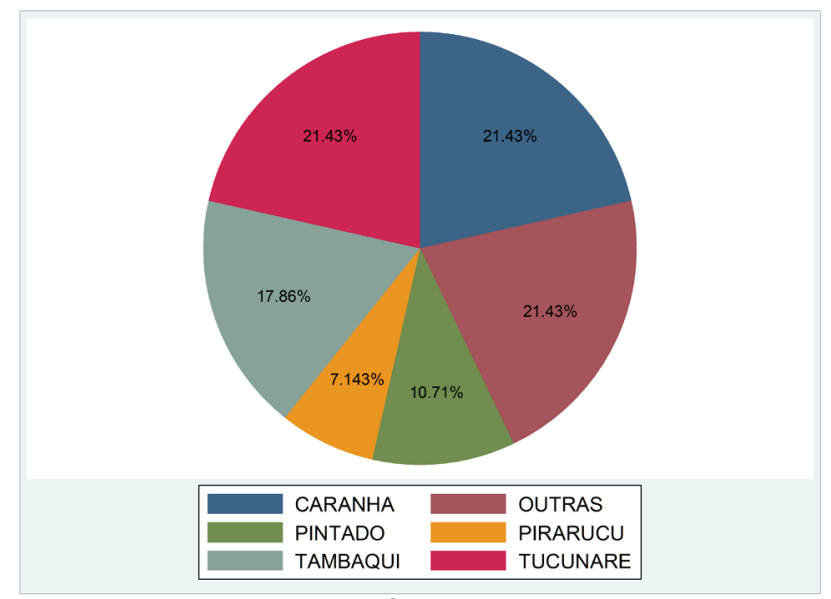

Figura 2 - Distribuição das preferências de espécie de peixes para alimentação entre participantes que estudam ou trabalham na área de pesca ou aquicultura. 
Tabela 3 - Espécies preferidas para consumo por nível de escolaridade.

\begin{tabular}{lcc}
\hline \hline Nível de escolaridade & Espécie & \% Preferência \\
\hline Ensino Fundamental & Caranha & 31,25 \\
Ensino Médio & Caranha & 14,06 \\
Ensino Superior & Tambaqui & 20,59 \\
Pós-graduação & Tucunaré & 42,86 \\
Mestrado & Tambaqui & 25,00 \\
Doutorado & Tucunaré & 33,33 \\
\hline \hline
\end{tabular}

correlação com a demanda por tecnologias. Mais uma vez, o pescado fresco como a forma de conservação de pescado foi a alternativa mais escolhida, mas, desta vez, com uma porcentagem ainda maior, $75 \%$. O mesmo pode ser observado para participantes que não são profissionais da área de pesca ou aquicultura. Isto é, pescado fresco foi a alternativa selecionada pela grande maioria dos participantes. Isso também pode ser visto na figura 4.

É importante analisar também as respostas dos participantes de acordo com o gênero, dado que homens e mulheres podem possuir diferentes preferências com relação à forma de conservação no momento da compra do pescado. A figura 5 apresenta as preferências para as mulheres para os homens.

O resultado mostra que as preferências entre os dois gêneros não possuem diferenças significativas, além de estarem semelhantes aos demais resultados com, mais uma vez, o pescado fresco sendo alvo da preferência da grande maioria dos participantes pesquisados.

Por fim, pode-se considerar as escolhas dos participantes por diferentes níveis de escolaridade. A tabela 4 apresenta os tipos de conservação de pescado que os participantes mais indicaram como preferidos para a compra. Observando a tabela pode-se verificar que, mais uma vez, o pescado fresco foi indicado como a forma de conservação mais comprada pra todos os níveis de escolaridade.

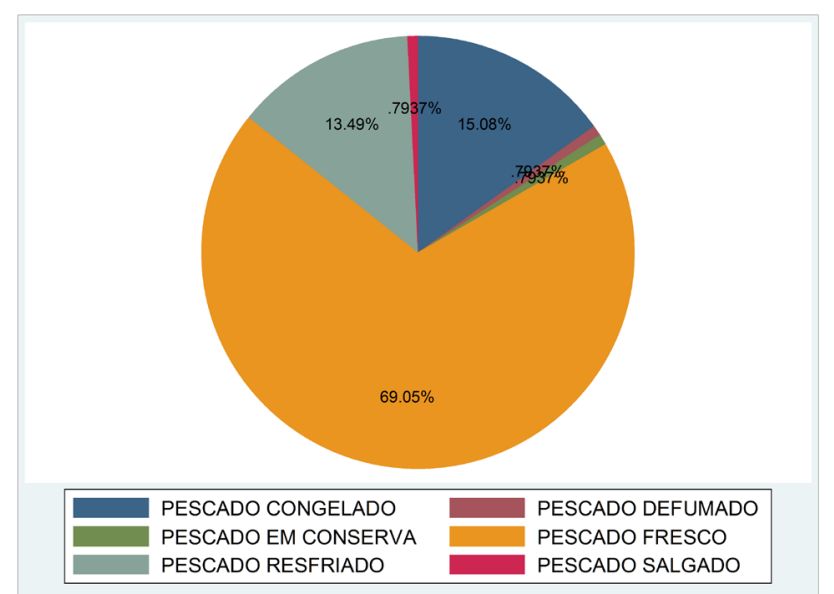

Figura 3 - Distribuição das formas de conservação mais compradas de pescado para todos os participantes do seminário

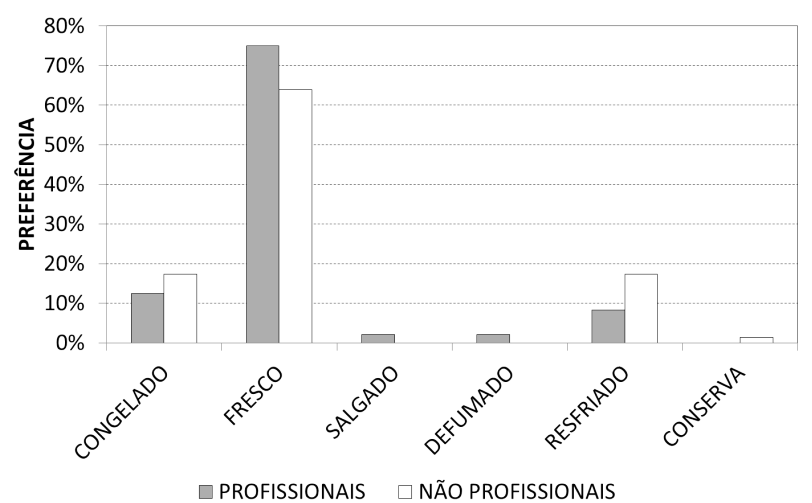

Figura 4 - Distribuição das formas de conservação mais compradas de pescado entre os profissionais e não profissionais da área.

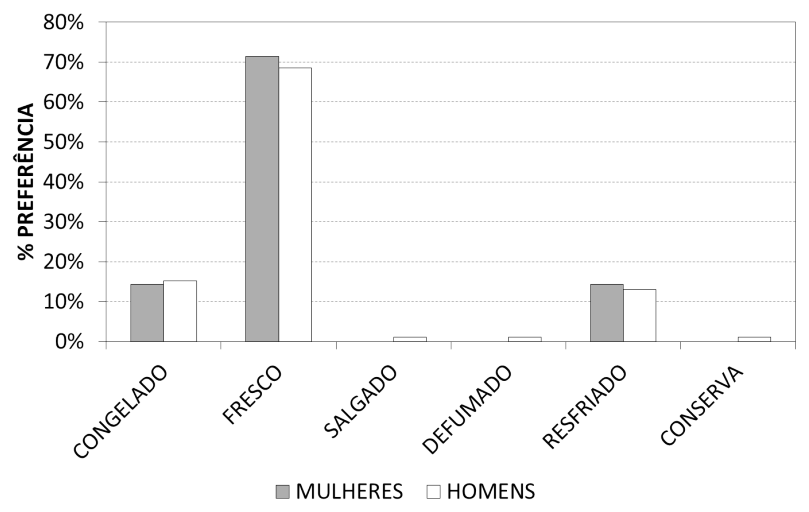

Figura 5 - Distribuição das formas de conservação mais compradas de pescado entre as mulheres e homens.

\section{Forma de apresentação de pescado considerada ideal para o dia-a-dia}

A Questão 6 dos questionários respondidos pelos participantes do seminário é "Em relação a forma de apresentação do pescado, qual você considera ideal para o dia-a-dia?". A figura 6 apresenta a distribuição das respostas observadas para todos os participantes que responderam este questionário, mostrando que a grande maioria dos participantes aponta o pescado Inteiro como a forma de apresentação de pescado mais indicada, com mais de $50 \%$ das respostas. Filé e Eviscerado foram, com 29,01\% e $11,45 \%$, respectivamente, as formas de apresentação com uma porcentagem também significativa na pesquisa. Por outro lado, Espalmado e Posta tiveram uma porcentagem de escolha baixa na pesquisa.

Tabela 4 - Tipo de conservação de pescado preferido por nível de escolaridade

\begin{tabular}{lcc}
\hline \hline Nível de escolaridade & Tipo de conservação & \% Preferência \\
\hline Ensino Fundamental & Pescado Fresco & 43,75 \\
Ensino Médio & Pescado Fresco & 62,50 \\
Ensino Superior & Pescado Fresco & 58,82 \\
Pós-graduação & Pescado Fresco & 42,86 \\
Mestrado & Pescado Fresco & 50,00 \\
\hline \hline
\end{tabular}




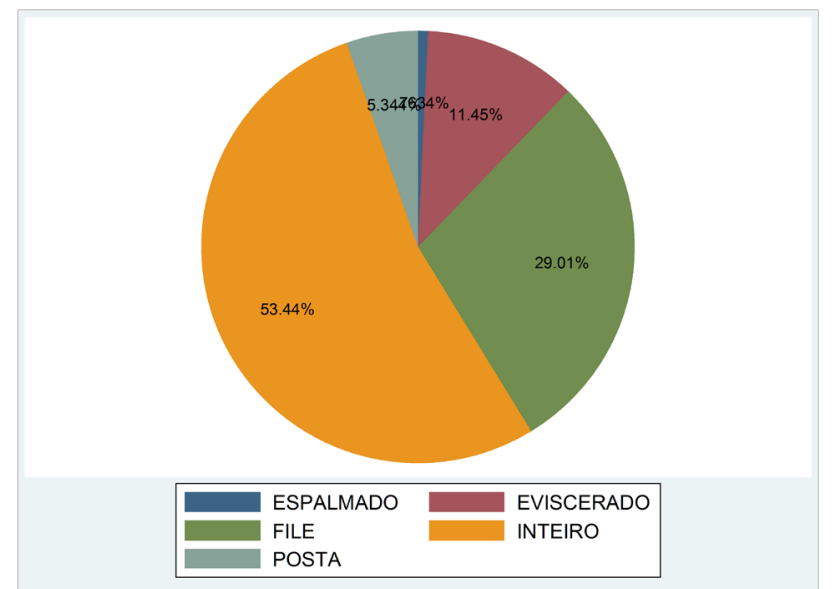

Figura 6 - Distribuição das formas de apresentação de pescado mais indicadas para todos os participantes do seminário.

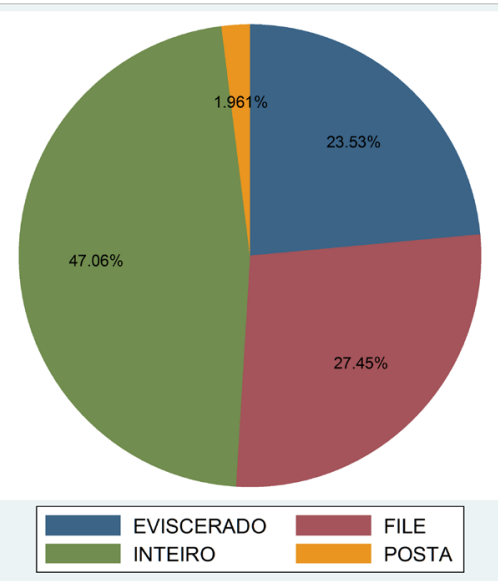

Figura 7 - Distribuição das formas de apresentação de pescado preferidas entre os profissionais da área.

Vale observar que há possibilidade de a forma de apresentação mais indicada pelos participantes ter sido o pescado inteiro devido ao fato dos indivíduos pesquisados não compreenderem exatamente as diferenças entre as alternativas de respostas fornecidas para esta questão. Mais especificamente, os entrevistados indicaram o pescado Inteiro, mas é muito provável que, dificilmente, preferem que este pescado seja comprado com as vísceras. Sendo assim, acredita-se que uma parte significativa dos entrevistados que respondeu Inteiro, poderia estar se referindo ao pescado eviscerado.

Quando se considera apenas as respostas dos profissionais da área, a distribuição das formas de apresentação segue conforme a figura 7. Como pode ser visto, este gráfico apresenta mudanças em relação à figura 6 , mostrando que a forma de apresentação Eviscerado teve um considerável aumento nas escolhas dos participantes. Este resultado vem de encontro com a hipótese levantada no parágrafo anterior, como profissionais da área possuem mais conhecimento sobre as formas de apresentação de pescado, há uma melhor identificação das alternativas

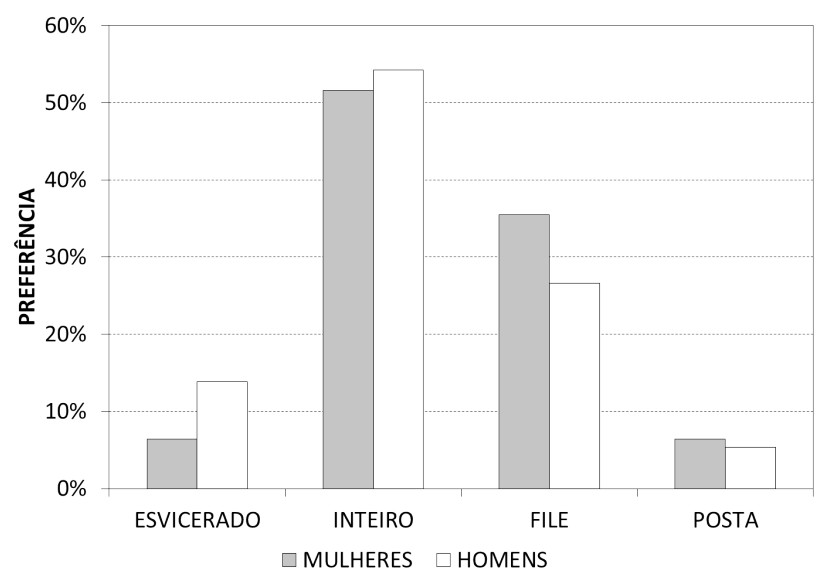

Figura 8 - Distribuição das formas de apresentação de pescado preferidas entre as mulheres e homens.

sugeridas e um melhor entendimento do que é um pescado eviscerado.

Assim como no caso da forma de conservação, é importante também analisar as respostas dos participantes para a forma de apresentação de acordo com o gênero, dado que homens e mulheres podem possuir diferentes preferências. A figura 8 mostra que as preferências entre os dois gêneros não possuem diferenças significativas, além de estarem semelhantes ao resultado geral, indicando que não há indícios de correlação entre o gênero e a forma de apresentação preferida.

Por fim, pode-se considerar as escolhas dos participantes por diferentes níveis de escolaridade. A tabela 5 apresenta as formas de apresentação de pescado que os participantes mais indicaram como preferidos para a compra. Observando a tabela pode-se verificar que o pescado fresco e o filé foram as formas de apresentação preferidas. Mais especificamente, pescado inteiro é a forma preferida entre as escolaridades mais baixas e filé é a forma de apresentação preferida entre os participantes com escolaridade mais alta.

\section{Local de compra do peixe para consumo}

A Questão 7 dos questionários respondidos pelos participantes do seminário é "Onde você compra/adquire o peixe para consumo?". A maioria dos participantes aponta mercado como o local escolhido para compras de peixe, com mais de $40 \%$ das res-

Tabela 5 - Forma de apresentação de pescado preferida por nível de escolaridade.

\begin{tabular}{lcc}
\hline \hline Nível de escolaridade & Forma de apresentação & \% Preferência \\
\hline Ensino Fundamental & Inteiro & 53,13 \\
Ensino Médio & Inteiro & 54,69 \\
Ensino Superior & Filé & 41,18 \\
Pós-graduação & Inteiro & 28,57 \\
Mestrado & Filé & 25,00 \\
Doutorado & Filé & 33,33 \\
\hline \hline
\end{tabular}


postas (figura 9). Pesca própria, feira e direto com o pescador foram, com $25,77 \%, 18,56 \%$ e $12,37 \%$, respectivamente, as formas de apresentação com uma porcentagem também significativa na pesquisa.

Quando se considera apenas as respostas dos profissionais da área (figura 10), a distribuição dos locais de compra apresenta mudanças, com o peixe vindo de pesca própria sendo a forma de aquisição de peixe mais apontada. Este resultado faz sentido, dado que uma parte considerável dos participantes da pesquisa é de pescadores.

Mais uma vez, é importante também analisar as respostas dos participantes para o local de compra de acordo com o gênero, dado que homens e mulheres podem possuir diferentes preferências. $O$ resultado mostra que as preferências entre os dois gêneros possuem algumas diferenças (figura 11), mas que são justificadas pelo fato de a maioria dos participantes pescadores serem do sexo masculino. Assim, os resultados mostram que as mulheres compram mais em mercados comparadas com os homens que, por

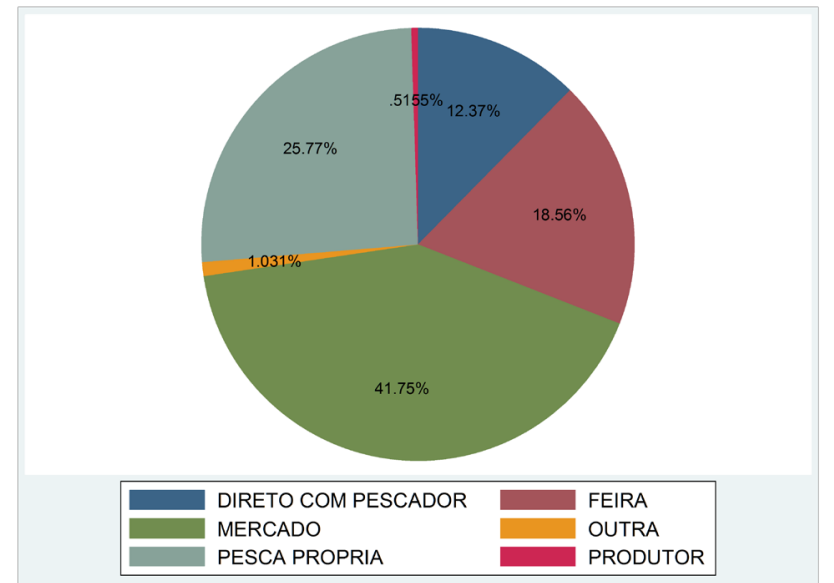

Figura 9 - Distribuição dos locais de compra de pescado mais indicados para todos os participantes do seminário.

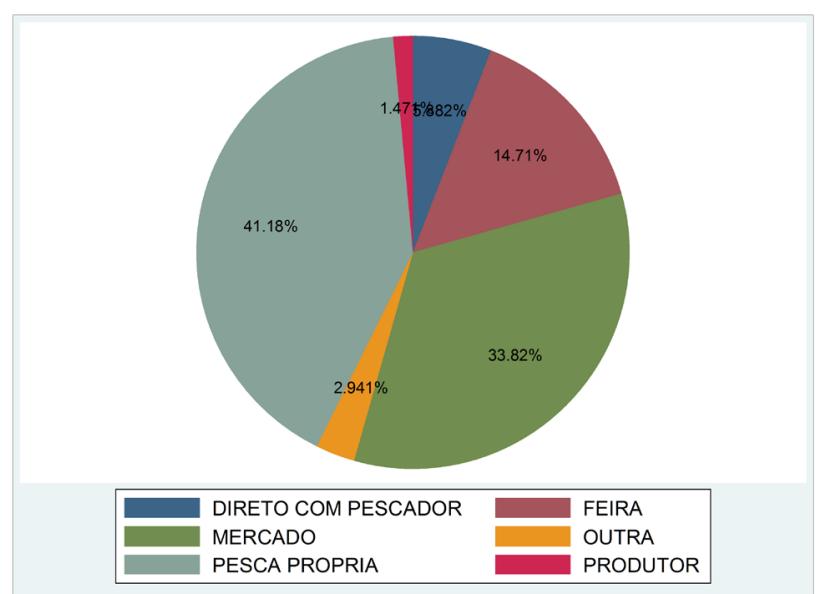

Figura 10 - Distribuição dos locais de compra preferidos entre os profissionais da área.

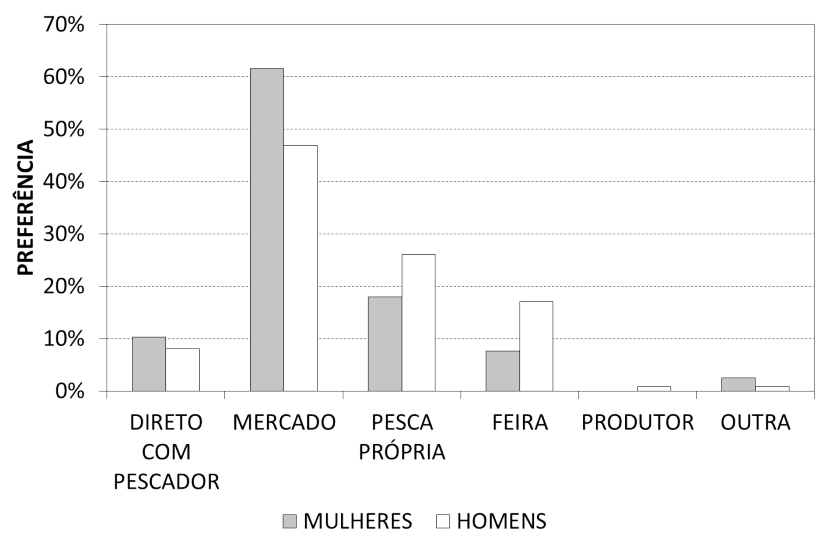

Figura 11 - Distribuição dos locais de compra de peixe preferidos entre as mulheres e homens.

sua vez, possuem um percentual relativamente maior de peixe advindo de pesca própria.

Pode-se considerar também as escolhas dos participantes por diferentes níveis de escolaridade. A Tabela 6 apresenta os locais de compra de peixe que os participantes mais indicaram como preferidos. Observando a tabela pode-se verificar que pesca própria foi a alternativa mais indicada apenas para os participantes com escolaridade mais baixa. Esse resultado vem de encontro com o fato de os pescadores, além de serem homens em sua maioria, possuírem na média escolaridade mais baixa. No geral, mercado continuou sendo a alternativa mais lembrada.

\section{Dificuldades no mercado de pescado}

A Questão 8 dos questionários respondidos pelos participantes do seminário é "Qual das opções abaixo você considera a principal dificuldade no mercado de pescado". A figura 12 apresenta a distribuição das respostas observadas para todos os participantes que responderam este questionário e mostra que altos preços e falta da espécie desejada são as dificuldades mais apontadas pelos participantes. Além disso, falta de qualidade, dificuldade no preparo e falta da apresentação desejada também foram dificuldades bastante lembradas pelos participantes.

Quando se consideram apenas as respostas dos profissionais da área (figura 13), não se observam mudanças significativas em relação à figura 12 , mostrando que não há evidente correlação entre as

Tabela 6 - Locais de compra de compra de peixe preferidos por nível de escolaridade.

\begin{tabular}{lcc}
\hline \hline Nível de escolaridade & Locais de compra & \% Preferência \\
\hline Ensino Fundamental & Pesca própria & 62,50 \\
Ensino Médio & Mercado & 46,88 \\
Ensino Superior & Mercado & 73,53 \\
Pós-graduação & Mercado & 71,43 \\
Mestrado & Mercado & 100,00 \\
Doutorado & Mercado & 100,00 \\
\hline \hline
\end{tabular}




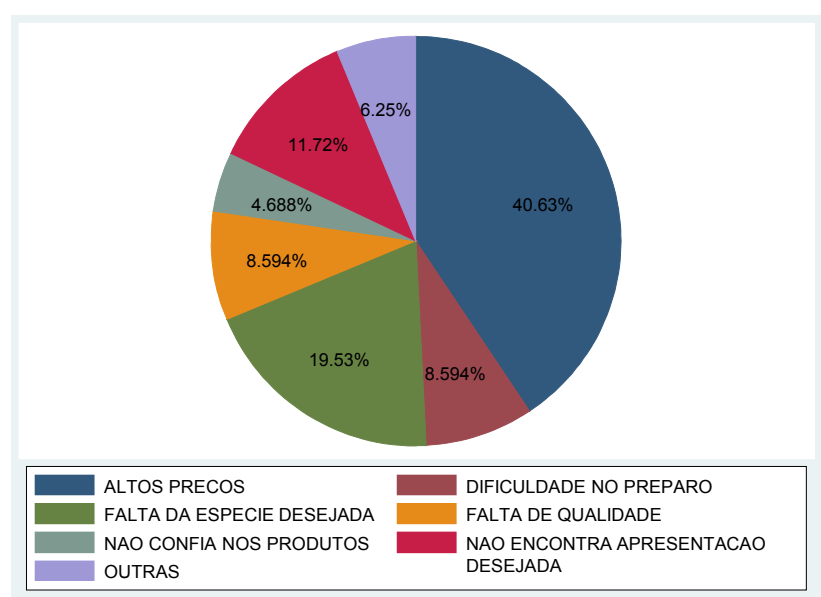

Figura 12 - Distribuição das dificuldades do mercado de pescado mais indicadas para todos os participantes do seminário.

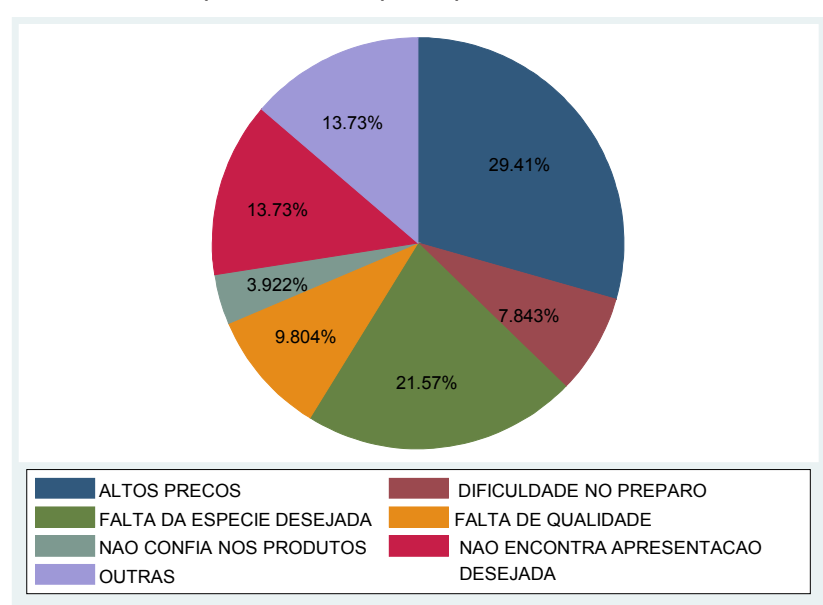

Figura 13 - Distribuição das dificuldades no mercado de pescado para os profissionais da área.

dificuldades de mercado e o fato de ser um profissional da área. Este resultado é interessante, pois mesmo as alternativas mais relacionadas a questões profissionais, como financiamento e transporte, não tiveram uma porcentagem mais elevada.

É importante também analisar as respostas dos participantes sobre as dificuldades encontradas no mercado de acordo com o gênero, dado que homens e mulheres podem possuir diferentes percepções. Entre

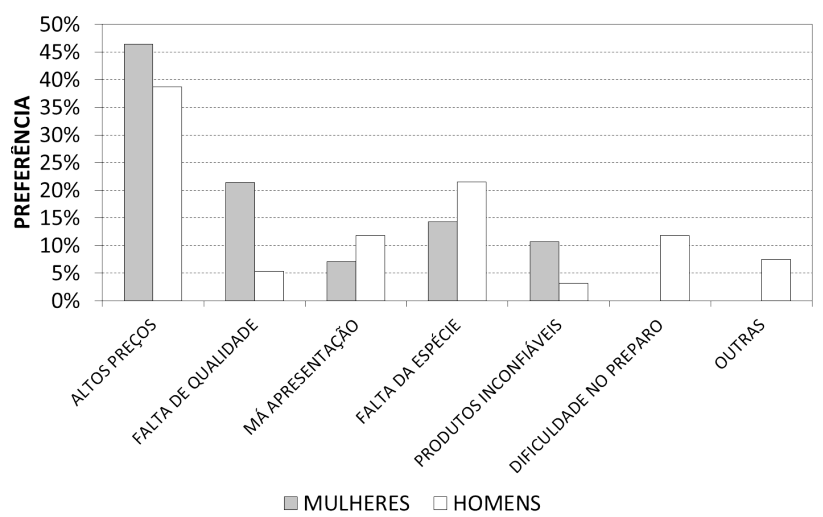

Figura 15 - Distribuição das dificuldades de mercado entre as mulheres e homens.
Tabela 7 - Dificuldade do mercado de pescado por nível de escolaridade.

\begin{tabular}{lcc}
\hline \hline Nível de escolaridade & Dificuldades & \% Preferência \\
\hline Ensino Fundamental & Falta da espécie desejada & 31,25 \\
Ensino Médio & Altos preços & 34,38 \\
Ensino Superior & Altos preços & 44,12 \\
Pós-graduação & Altos preços & 38,10 \\
Mestrado & Altos preços & 50,00 \\
\hline \hline
\end{tabular}

as mulheres, o preço alto do peixe é a maior dificuldade encontrada com quase $50 \%$ das indicações (figura 15). Outra dificuldade com alto percentual foi falta de qualidade, mostrando a maior preocupação feminina para esta questão, já que entre os homens a falta de qualidade ficou longe de ser uma das dificuldades mais apontadas. Para os homens, preço alto também foi a maior dificuldade apontada, mas, em segundo lugar, se encontra a falta de espécie desejada.

Por fim, pode-se considerar as escolhas dos participantes por diferentes níveis de escolaridade. A Tabela 7 apresenta as dificuldades no mercado de pescado que os participantes mais indicaram. Observando a tabela pode-se verificar que apenas para participantes com ensino fundamental a falta de espécies desejadas foi o principal problema do mercado. Para os demais níveis mais altos de escolaridade, os altos preços foram apontados como a maior dificuldade no mercado de pescado.

\section{DISCUSSÃO}

A presente pesquisa entrevistou 162 pessoas durante a realização do seminário nacional "Caiu na Rede é Lucro", realizado em Palmas - TO. O evento, que marcou o início dos trabalhos do sistema FAET/ CNA para incentivo à produção de pescado, contou com a presença de aproximadamente 300 participantes entre profissionais e não profissionais da área de pesca e aquicultura.

Os objetivos deste trabalho foram: identificar o público participante do seminário, diagnosticar quais são as principais demandas para o estado do Tocantins de tecnologia e capacitação a serem desenvolvidas na área de pesca e aquicultura e entender melhor as preferências do consumidor tocantinense de pescado no que se refere a locais de compra, espécies preferidas, formas de apresentação e tipos de conservação.

Com relação às espécies de peixes preferidas para consumo, Tucunaré e caranha foram as mais apontadas. Caranha foi a espécie mais escolhida entre os participantes de escolaridade mais baixa, mas em compensação, quase não foi lembrada entre as muIheres. Essa diferenciação pode vir da dificuldade de identificação das espécies preferidas Muitas vezes há 
confusão entre as espécies de peixes redondos e isso faz com que esses padrões identificados no consumo estejam com esse viés. Tambaqui, embora não tenha sido a mais lembrada para consumo, é uma das espécies mais trabalhadas entre os profissionais da área.

O trabalho também investigou sobre as preferências de conservação, apresentação e locais de compra de pescado. Pescado fresco foi a forma de conservação escolhida pela grande maioria dos participantes, independente de sexo, escolaridade ou atuação profissional. Já para apresentação do pescado, o pescado inteiro foi a forma preferida, embora acredite-se que essa preferência seja decorrência da não distinção entre pescado inteiro e pescado eviscerado entre os participantes. Para os níveis de escolaridade mais altos o pescado em forma de filé foi apontado como o preferido.

Os locais de compra mais utilizados entre os participantes foram o mercado e o peixe advindo de pesca própria. Esse resultado é devido à grande participação de pescadores no seminário. Se for considerado o público em geral, a grande maioria compra o peixe nos mercados. Por fim, quando questionados sobre as principais dificuldades encontradas no mercado de pescado, no geral, os participantes apontaram que os altos preços são o principal gargalo do setor.

Sendo assim, o presente trabalho buscou esclarecer e indicar alguns pontos importantes sobre as demandas na área de pesca e aquicultura. Tanto com demandas em relação ao consumo final quanto com demandas sobre tecnologias e capacitações foram levantadas necessidades e preferências tanto de profissionais da área quanto de participantes que são apenas consumidores. Fica então a oportunidade para explorar estes resultados, direcionando as pesquisas da área e melhor atendendo o público consumidor quanto às suas preferências.

\section{CONCLUSÃO}

Com os resultados apresentados pode-se tirar algumas conclusões sobre o hábito de consumo do público consultado. Primeiramente, a preferência do consumidor por pescado fresco e inteiro mostra que as pessoas ainda não estão habituadas a comprar pescado com níveis mais avançados de processamento e maior valor agregado. Isso pode ser justificado pela cultura do consumidor de escolher o melhor modo de preparo de seu alimento, buscando preços mais baixos.

Deve-se observar também que os mercados foram os lacais mais apontados como preferidos para a compra do pescado. Assim, se por um lado os consumidores indicaram preferir os produtos menos processados, por outro lado preferem a comodidade de comprar em mercados, que são locais com maiores opções de outras mercadorias e demais comodidades para a compra.

O preço também tem papel importante na escolha do consumidor. Além de preferir comprar em mercados, que, em média, apresentam preços mais baixos para os mesmos produtos, foi indicado que o maior gargalo da cadeia produtiva do pescado são justamente os preços. Esse resultado indica que, no Brasil, um dos motivos do baixo consumo de pescado comparado com carnes advindas de outros animais pode ser devido aos altos preços, mais até do que a dificuldade de preparo dos produtos.

\section{REFERÊNCIAS}

FAO. 2012. The State of World Fisheries and Aquaculture. FAO Fisheries and Aquaculture Department. Rome, Italy.

Maciel, E. S. 2011. Perspectiva do consumidor perante produto proveniente da cadeia produtiva de tilápia do Nilo rastreada (Oreochromis niloticus) consumo de pescado e qualidade de vida. Tese de Doutorado. Centro de Energia Nuclear na Agricultura, Universidade de São Paulo. 299p.

Ostrensky, A; Boerger, W. A.; Chammas, M. A. 2008. Potencial para o desenvolvimento da aquicultura no Brasil. In: Ostrenky, A; Borghetti, J. R.; Soto, D. (Orgs.) Aquicultura no Brasil: o desafio é crescer. SEAP-FAO. 27-72pp.

Roppa, L. 2009. Perspectivas da produção mundial de carnes, 2007 a 2015. In: ergomix.com: Pecuária de corte: artigos técnicos. Disponível em: http:// pt.engormix.com/member_login.aspx?referer=yes.

Sidonio L.; Cavalcanti, I.; Capanema, L.; Morch, R.; Magalhães, G.; Lima, J.; Burns, V.; Alves Junior, A. J.; Mungioli, R. 2012. Panorama da aquicultura no Brasil: desafios e oportunidades. BNDES Setorial 35: $421-463$.

Sonoda, D. Y. 2007. Demanda por pescados no Brasil entre 2002 e 2003. Tese de Doutorado. Escola Superior de Agricultura Luiz de Queiroz, Universidade de São Paulo. 118p.
Submetido: Janeiro/2013 Revisado: Dezembro/2013 Aceito: Dezembro/2013 


\section{APÊNDICE}

\section{Questões aplicadas}

1) Em qual município reside?:

UF:

2) Escolaridade:
a) Ensino Fundamental
d) Pós-graduação
b) Ensino Médio Completo
e) Mestrado
c) Ensino Superior Completo
f) Doutorado

3) Qual é a espécie de peixe preferida para alimentação?

4) Você trabalha/estuda com peixe? Sim Não.

5) Em relação à forma de conservação, qual das opções é a mais comprada por você?
a) Pescado Fresco
d) Pescado Salgado
b) Pescado Congelado
e) Defumado
c) Pescado Resfriado
f) Em Conserva

6) Em relação a forma de apresentação do pescado, qual você considera ideal para o dia-a-dia?
a) Posta
d) Espalmado
b) Filé
e) Eviscerado
c) Inteiro
f) Outra:

7) Onde você compra/adquire o peixe para consumo?
a) Mercado
d) Pesca própria
b) Feira
e) Outra:
c) Direto com pescador

8) Qual das opções abaixo você considera a principal dificuldade no mercado de pescado?
a) Altos preços
e) Não confia nos produtos
b) Falta da espécie desejada f) Falta de qualidade
c) Dificuldade no preparo g) Outra:
d) Não encontra a apresentação desejada
9) Sexo: $\square$ Masculino $\square$ Feminino 